\title{
Neue Empfehlungen zur Säuglingspflege
}

\author{
Prof. Dr. med. Dietrich Abeck, Dermatologe, München
}

\section{Nach aktualisierten Empfehlungen zur Pflege der Säuglingshaut sollte nach der Geburt die Käseschmiere möglichst auf der Haut belassen werden. Gefolgt von regelmäßigem Eincremen kann dies zur Stärkung der Hautbarriere beitragen.}

Seit April 2016 gibt es eine aktualisierte und erweiterte Empfehlung zur Reinigung und Pflege der Säuglingshaut [1], basierend auf den Round table-Empfehlungen von 2009 [2]: Die Dermatologen der Berliner Charité aktualisierten und ergänzten gemeinsam mit europäischen Kollegen die Empfehlungen - auf Basis neuer Studiendaten, die sich vor allem mit der Reinigung des Säuglings direkt nach der Geburt befassen sowie mit der Fragestellung, ob eine Pflege der Säuglingshaut von Geburt an den Aufbau der noch unreifen Hautbarriere unterstützen kann.

\section{Vernix caseosa als natür- liche Hautschutzcreme}

Ein besonderes Gewicht der neuen Empfehlungen liegt nun auf der Käseschmiere (lat. Vernix caseosa): Die natürliche „Hautschutzcreme“ aus körpereigenen Fetten, fettähnlichen Substanzen und Aminosäuren (Protein-Bausteinen), die sich etwa in der 17. SSW auf der Haut ungeborener Babys bildet, bewahrt das Kind im Mutterleib vor einem allzu intensiven Kontakt mit dem Fruchtwasser und nach der Geburt vor Austrocknung sowie Infektionen. Zudem verfügt sie über eine intensive Pflegewirkung. Zusätzlich bewahrt die Käseschmiere das Baby nach der Geburt vor dem Auskühlen.

Deshalb wird in der neuen Empfehlung von 2016 folgendes Vorgehen beschrie- ben: „Die vom Fruchtwasser und dem Blut der Mutter feuchte Haut sollte in erster Linie zügig getrocknet werden, um einen Abfall der Körpertemperatur zu verhindern. Im Unterschied zu den Empfehlungen von 2009 sollte das Neugeborene deshalb nicht mehr mit Wasser abgewaschen, sondern nur mit einem trockenen Handtuch vorsichtig abgewischt werden. Lediglich starke Verunreinigungen sollten weiterhin mit Wasser entfernt werden. Gleichzeitig bleibt auf diese Weise die Käseschmiere (Vernix caseosa) erhalten (...). Bei der ersten Reinigung ist es deshalb sinnvoll, die Käseschmiere in die Haut des Neugeborenen einzumassieren“ [3].

Die neuen Erkenntnisse um die schützenden Wirkstoffe in der Käseschmiere standen Pate auch für die Entwicklung neuer Pflegeprodukte für Neugeborene, um die positiven Effekte auch nach der Geburt nutzen zu können.

\section{Neue Erkenntnisse}

\section{Das erste Bad ist schon bei Stabilisierung der Körpertemperatur erlaubt}

Sobald sich die Körpertemperatur stabilisiert hat, dürfen Neugeborene das erste Mal gebadet werden, auch wenn der Nabelschnurrest noch nicht abgefallen ist. Diese Stabilisierung tritt gewöhnlich in den ersten vier Wochen ein.
Beim ersten Bad ist für das Pflegepersonal empfehlenswert, Handschuhe zu benutzen, um sich nicht mit eventuellen Keimen der Mutter zu kontaminieren. In der früheren Empfehlung wurde dies als weniger relevant erachtet, da die Kinder erst einige Tage später gebadet und zudem bei der Geburt abgewaschen wurden. Somit waren keine mütterlichen Rückstände von der Geburt (Fruchtwasser, Blut etc.) mehr am Körper des Kindes erkennbar.

\section{Baden mindestens zwei- bis dreimal pro Woche empfohlen}

Das Baden des Säuglings ist wesentlich schonender für die Haut als das Waschen mit dem Lappen. Deshalb ist es unbedingt zu bevorzugen. Als Teil der Abendroutine, mindestens zwei- bis dreimal pro Woche, kann ein Bad das Schlafverhalten verbessern und positiven Einfluss auf die Eltern-Kind-Bindung ausüben. Auch das Tastempfinden des Säuglings wird durch ein Bad stimuliert.

Dabei ist Folgendes zu beachten:

- Die empfohlene Raumtemperatur liegt nun zwischen 21 und $24^{\circ} \mathrm{C}$ (frühere Empfehlung: $22^{\circ} \mathrm{C}$ )

- Die Wassertemperatur ist zwischen 37 und $37,5^{\circ} \mathrm{C}$ einzustellen.

- Die optimale Badedauer liegt bei maximal fünf bis zehn Minuten.

- Badewanne und -spielzeug sollten sauber gehalten, müssen aber nicht desinfiziert werden. 
Speziell für die Babyhaut entwickelte Reinigungspräparate werden gut toleriert und sind dem Waschen mit Wasser nicht unterlegen. Empfohlen wird, statt Seife die milderen Syndets (synthetische, waschaktive Substanzen) mit leicht saurem pH-Wert (in der Regel 5,5) zu nehmen, da diese die normale Reifung der Hautbarriere nicht negativ beeinflussen. Hier sollten allerdings ausschließlich Reinigungsprodukte zum Einsatz kom- men, deren Inhaltsstoffe bekannt und für die Anwendung bei Säuglingen zugelassen sind.

\section{Hautpflege für Neugeborene mit wirkstofffreier Feuchtig- keitscreme}

Eine wesentliche Neuerung in den Empfehlungen ist, Neugeborene konsequent mit wirkstofffreier Feuchtigkeitscreme einzucremen, um die Hautbarriere zu stabilisieren. Es sollte eine Emulsion, also eine Creme oder Lotion, verwendet werden. Neuere Studien belegen, dass eine gezielte Hautpflege von Anfang an eine gesunde und widerstandsfähige Hautschutzbarriere fördert (s. Kasten). Zu empfehlen ist, dass damit ab der 2. oder 3. Lebenswoche begonnen wird. Die empfindliche Haut des Neugeborenen

\section{Aktuelle Studien: Natürliche Hautbarriere erhalten}

Die Barrierefunktion ist eine der wichtigsten Eigenschaften der Haut. Bei Säuglingen jedoch ist noch keine ausreichende Menge an Hautlipiden gebildet worden, die den Zusammenhalt der Hornzellen in der Hornschicht gewährleisten. Deshalb ist die Haut durchlässiger und kann Wasser weniger stark binden. Das macht sie empfindlicher für Schadstoffe von außen und lässt den transepidermalen Wasserverlust (TEWL), also den Feuchtigkeitsverlust über die Haut, steigen. Ebenso produzieren die Talgdrüsen noch nicht ausreichend Fett für einen schützenden Fett-/Feuchtigkeitsfilm.

Inzwischen belegen Studien, dass durch gezielte Hautpflege von Anfang an eine gesunde und widerstandsfähige Hautschutzbarriere aufgebaut werden kann. Das wiederum sorgt für einen ausgeglichenen Feuchtigkeitshaushalt und stärkt den Eigenschutz der Haut gegenüber schädlichen Umwelteinflüssen, zu denen auch Allergene und sensibilisierende Stoffe gehören, die Allergien auslösen können. Hier ein kurzer Überblick über die wichtigsten Studien:

- Basispflege mit Babylotion oder Sonnenblumenöl verbessert Hautbarriere bei gesunden Säuglingen nachweislich. Tägliche Basispflege der gesunden Säuglingshaut: 50 gesunde Reifgeborene wurden ab dem vierten Lebenstag über fünf Wochen drei Mal die Woche entweder mit einer Pflegelotion für Säuglinge mit hohem Anteil Sonnenblumenöl oder mit reinem Sonnenblumenöl eingecremt [4]. Es wurden die Hautparameter dokumentiert sowie der allgemeine Zustand der Haut bewertet: Die Hautbarrierefunktion entwickelte sich mit der täglichen Hautpflege physiologisch normal, es wurde aber eine Verbesserung der Hautfeuchtigkeit dokumentiert sowie die erhoffte Abnahme des Wasserverlustes. Interessant war, dass es zu Schwankungen der Sebum-Werte (Hautfett) kam. Ein tendenzieller Anstieg unter der Babylotion und ein Absinken unter Sonnenblumenöl konnte beobachtet werden. Die Hautbeschaffenheit (Neonatal Skin Condition Score) verbesserte sich in beiden Gruppen, beide Pflegeprodukte wurden sehr gut vertragen.

- Olivenöl irritiert die Haut. Bei einer Pflege mit Olivenöl dagegen steigt der Feuchtigkeitsverlust über die Haut sogar an: Eine Studie von M. Cork und S. G. Danby führte kürzlich zu einem klaren Umdenken in der Anwendung von Olivenöl bei Säuglingen [5]. Die Autoren untersuchten den Effekt von hoch gereinigtem Olivenöl auf der Haut erwachsener Probanden mit oder ohne Veranlagung zu atopischer Dermatitis. Dokumentiert wurden die Beschaffenheit der Hautbarriere sowie eventuell auftretende Hautirritationen. Das Ergebnis: Olivenöl trocknete die Haut sogar aus, es kam zu leichten Hautirritationen.

- Tägliche Pflege von Geburt an senkt das Risiko, eine atopische Dermatitis zu entwickeln, um $\mathbf{5 0} \%$. Ziel der amerikanischen Untersuchung war es, aufzuzeigen, ob eine Verbesserung der Hautbarriere bei Neugeborenen möglicherweise eine sinnvolle Strategie sein könnte, um eine atopische Dermatitis (AD) bei Risikokindern zu reduzieren [6]. Die randomisierte kontrollierte Studie schloss 124 Kinder aus den USA und UK ein, behaftet mit einem hohen Risiko für AD (seitens Eltern oder mindestens einem der Geschwister). Die Eltern waren instruiert, mindestens einmal täglich ab dem 22. Lebenstag ihr Baby am ganzen Körper mit Emulsion einzucremen. In der Kontrollgruppe durfte nicht gecremt werden. Das Ergebnis: Die tägliche Pflege reduzierte die kumulative Inzidenz einer atopischen Dermatitis im Alter von sechs Monaten von $43 \%$ bei der Kontrollgruppe auf $23 \%$ in der Emollentiengruppe. Dies entspricht einer relativen Risikominderung um 50\%.

- Frühe Pflege führt zu signifikanter Verbesserung der Stratum Corneum-Hydratation. Eine japanische Arbeitsgruppe ging der Frage nach, ob eine frühe Pflege der Säuglingshaut zur Stärkung der Hautbarriere eine atopische Dermatitis verhindern oder verzögern kann [7]. Die Forscher ließen 59 Säuglinge einer Hochrisikogruppe für AD (definiert durch AD bei Eltern und/oder Geschwistern) täglich mit Emulsionen eincremen. Das Ergebnis: Durch die tägliche Hautpflege mit Emollentien traten in 23\% der Fälle weniger Ekzeme auf, am oberen Bein konnten in der Gruppe der eingecremten Säuglinge statistisch signifikante Verbesserungen der Stratum Corneum-Hydratation gemessen werden. 
sollte künftig mindestens zweimal wöchentlich dünn eingecremt werden, besonders nach dem Baden. Denn diese zusätzliche Pflege sorgt für einen ausgeglichenen Feuchtigkeitshaushalt und stärkt den Eigenschutz der Haut gegenüber schädlichen Umwelteinflüssen, zu denen auch Allergene und sensibilisierende Stoffe, die Allergien auslösen können, gehören.

Um den natürlichen und gleichzeitig höchst effizienten Schutz der Käseschmiere auf gutem Wirkungslevel fortzuführen, kann es hilfreich sein, die besondere Zusammensetzung der Käseschmiere zu entschlüsseln, um sie mit pflanzlichen Stoffen nachzuempfinden. Dies bedeutet nicht nur, einzelne Bestandteile wie zum Beispiel spezielle Fettsäuren oder Aminosäuren zu identifizieren, sondern eine Gesamtformulierung zu finden, die die Wirkeigenschaften der Vernix caseosa übernehmen kann.

\section{Zusammenfassung der Empfehlungen}

- Neugeborene sollten nach der Geburt lediglich bei starken Verunreinigungen mit reinem Wasser gewaschen, ansonsten nur mit einem trockenen Handtuch vorsichtig abgewischt werden.

- Vorhandene Käseschmiere sollte einmassiert werden.

- Das erste Baden kann nach Stabilisierung der Körpertemperatur und nicht mehr vorhandener Käseschmiere erfolgen. Hebammen/Pflegekräfte sollten dabei aufgrund eventuell vorhandener mütterlicher Keime Handschuhe tragen.

- Baden ist wesentlich schonender für die Babyhaut als Waschen mit dem Lappen, darum kann der Säugling mindestens 2-3 mal wöchentlich für maximal 5-10 Minuten gebadet

\section{In eigener Sache: Infoportal von Hautspezialisten}

Das Infoportal für Hautkrankheiten http://derma.plus bietet von Experten verfasste Texte zu allen geläufigen Hautkrankheiten. Hinter derma.plus steht ein Team von Professoren, die die Qualität der Informationen sicherstellen. Die Beiträge auf derma.plus geben den aktuellen Wissens- und Behandlungsstand wieder und werden stetig den neuen wissenschaftlichen Erkenntnissen angepasst. Auch die langjährigen Erfahrungen aus der Praxis der Experten fließen in die Empfehlungen und Tipps zur Behandlung mit ein.

werden. Säuglingsbadezusätze haben eine positive Wirkung auf die Babyhaut.

- Nach dem Baden sollte jeder Säugling am ganzen Körper mit einer wirkstofffreien Feuchtigkeitscreme eingecremt werden, um die Hautbarriere zu stabilisieren und der Entstehung von Neurodermitis vorzubeugen.

- An den anderen Tagen erfolgt einmal täglich zusätzlich zur regelmäßigen Gesäßreinigung eine vorsichtige Reinigung des Gesichts, der Ohren und der Hautfalten. Das Gesicht ist mit einer Babycreme zu pflegen.

- Zur Reinigung sind waschaktive Substanzen mit einem leicht sauren pH-Wert zu verwenden.

- Nach der Reinigung des Gesäßes sollte der Po des Kindes mit einer Babycreme geschützt werden.

- Es sollten ausschließlich Reinigungsund Pflegeprodukte verwendet werden, die für die Anwendung bei Säuglingen zugelassen sind.

\section{Literatur}

1 Blume-Peytavi U et al. Pediatr Dermatol 2016; 33: 311-321

2 J Eur Acad Dermatol Venereol 2009; 23 (7): 751-9

3 https://derma.plus/journal/hautpflegebei-saeuglingen/, letzter Zugriff 15.8.2016

4 Danby SG et al. Effect of olive and sunflower seed oil on the adult Skin barrier: Implications for neonatal skincare. Pediatr Dermatol 2013; 30 (1): 42-50

5 Kanti V et al. Intern J Dermatol in preparation

6 Simpson EL et al. Emollient enhancement of the skin barrier from birth offers effective atopic dermatitis prevention. J Allergy Clin Immunol 2014; 134: 818-823

7 Horimukai K et al. Application of moisturizer to neonates prevents development of atopic dermatitis. J Allergy Clin Immunol 2014; 134: 824-30

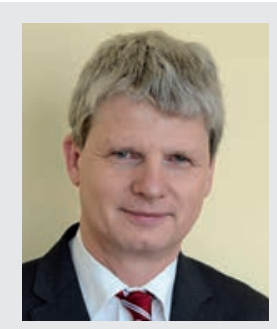

Prof. Dr. med. Dietrich Abeck Gemeinschaftspraxis für Dermatologie und Venerologie - Allergologie Konsiliararzt Renatastraße 72 80639 München E-Mail: professorabeck@mytum.de 